\title{
UTILIZAÇÃO DA MADEIRA DE Eucalyptus benthamii NA PRODUÇÃO DE PAINÉIS DE PARTÍCULAS ORIENTADAS (OSB)
}

\author{
Luciane Gorski ${ }^{1}$, Alexsandro Bayestorff da Cunha ${ }^{2 *}$, Polliana D’Angelo Rios ${ }^{2}$, Rosilani Trianoski ${ }^{3}$, \\ Morgana Cristina França ${ }^{1}$, Caio Cesar Faedo de Almeida ${ }^{1}$, Bruna Laís Longo ${ }^{1}$ \\ ${ }^{1}$ Universidade do Estado de Santa Catarina, Curso de Mestrado em Engenharia Florestal, Lages, Santa Catarina, Brasil - \\ lu_gorski@hotmail.com; morganaa_franca@hotmail.com, caio-almeida@ florestal.eng.br; bruna.lais@ florestal.eng.br \\ ${ }^{2}$ Universidade do Estado de Santa Catarina, Centro Agroveterinário, Departamento de Engenharia Florestal, Lages, Santa Catarina, \\ Brasil -*a2abc@cav.udesc.br; a2pdr@cav.udesc.br \\ ${ }^{3}$ Universidade Federal do Paraná, Departamento de Engenharia e Tecnologia Florestal, Curitiba, Paraná, Brasil - \\ rosillani@gmail.com
}

Recebido para publicação: 25/04/2015 - Aceito para publicação: 03/07/2015

O objetivo foi produzir painéis OSB com partículas de E. benthamii e mix de Pinus taeda e Pinus elliottii. O delineamento experimental envolveu cinco tratamentos compostos por painéis com três camadas de partículas e densidade nominal de $0,65 \mathrm{~g} / \mathrm{cm}^{3}$. Foram aplicados $6 \%$ de resina fenol formaldeído e $1 \%$ de emulsão de parafina. O ciclo de prensagem foi de 8 minutos, temperatura de $180^{\circ} \mathrm{C}$ e pressão específica de $40 \mathrm{kgf} / \mathrm{cm}^{2}$. Os ensaios foram realizados de acordo com a norma ASTM D1037 (1993) e a DIN 52362 (1982), sendo os resultados avaliados por meio da Análise de Variância e Teste de Scott-Knott. Como resultados, observou-se que as melhores composições foram obtidas no tratamento composto exclusivamente por partículas de Pinus spp e no tratamento com partículas de Pinus spp na camada central e E. benthamii nas camadas externas. Foi verificado potencial do Eucalyptus benthamii para a produção de painéis OSB quando misturada com partículas de $P$. taeda e $P$. eliiottii, tendo em vista que em painéis puros apresentou resultados insatisfatórios. Palavras-chave: Painéis de madeira; OSB; Eucalyptus sp e Pinus spp; Propriedades tecnológicas.

\begin{abstract}
Use of Eucalyptus benthamii wood in the production of Oriented Strand Boards (OSB). This research aimed to produce OSB with particles of E. benthamii and mix of Pinus taeda and Pinus elliottii. The experimental plan involved five treatments composed of panels with three layers of particles and nominal density of $0,65 \mathrm{~g} / \mathrm{cm}^{3}$. The resin phenol formaldehyde and the wax sizing were applied in the amount of $6 \%$ and $1 \%$, respectively. The pressing cycle was 8 minutes, temperature of $180{ }^{\circ} \mathrm{C}$ and specific pressure of $40 \mathrm{kgf} / \mathrm{cm}^{2}$. The assays were performed according to standard ASTM D1037 (1993) and DIN 52362 (1982) and the results were evaluated by Analysis of Variance and Scott-Knott Test. As result, it was observed that the best compositions are obtained in the treatment composed exclusively of Pinus spp particles and in the treatment with particles of Pinus spp in the core layer and the E. benthamii in the outer layers. It was verified potential of Eucalyptus benthamii for the production of OSB when mixed with particles of $P$. taeda and $P$. eliiottii, considering that in pure panels it showed unsatisfactory results.

Keywords: Wood panels; OSB; Eucalyptus sp and Pinus spp; Technological properties.
\end{abstract}

\section{INTRODUÇÃO}

O setor de produtos florestais é basicamente composto pelos segmentos de madeira em tora, madeira serrada, pasta de madeira, papel e painéis de madeira. Dentro desta última classe, de acordo com Del Menezzi (2004) são produzidos compensados, painéis de partículas aglomeradas (Medium Density Particleboard - MDP), painéis de fibra de média (Medium Density Fiberboard - MDF) e alta densidade (High Density Fiberboard HDF), painéis isolantes e painéis de partículas orientadas (Oriented Strand Board - OSB).

O OSB está no mercado mundial desde o início da década de 80, sendo considerado como a

FLORESTA, Curitiba, PR, v. 45, n. 4, p. 865 - 874, out. / dez. 2015.

Gorski, L. et al.

ISSN eletrônico 1982-4688 / ISSN impresso 0015-3826

DOI: $10.5380 /$ rf.v45i4.36280 
segunda geração dos painéis Waferboard. Os painéis OSB são produzidos a partir de partículas finas e longas, denominadas "strands", as quais são unidas umas as outras, pela ação da resina, pressão e altas temperaturas. Suas partículas são orientadas em número ímpar de camadas, geralmente três, sendo a camada interna posicionada perpendicularmente às externas (MALONEY, 1993).

A produção nacional atual de OSB utiliza predominantemente madeira de Pinus. No entanto, com a crescente demanda desse produto e, sendo a madeira dessa espécie utilizada para diversas outras finalidades, seu estoque pode não ser suficiente, sendo necessária a busca de novas espécies que possam se adequar ao processo (CASTRO et al., 2012). De acordo com Gouveia et al. (2003), a utilização de diferentes espécies e misturas na fabricação de painéis é descrita em diversas pesquisas. Isto, porque, basicamente, os painéis de partículas orientadas podem ser produzidos com diversas espécies, misturas de espécies ou diferentes estruturas de camadas.

Pode-se citar algumas pesquisas recentes com painéis OSB formados por diferentes espécies de rápido crescimento; Iwakiri et al. (2004) com seis espécies de Eucalyptus: E. maculata, E. dunnii, E. grandis, E. citriodora, E. tereticornis e E. saligna; Cabral et al. (2006) com E. grandis, E. urophylla, E. cloeziana e Pinus elliottii e Castro et al. (2012) com Pinus oocarpa e E. grandis.

Como a região sul do Brasil apresenta inverno rigoroso, uma espécie que vem se destacando em função do rápido crescimento e da tolerância á geadas é o Eucalptus benthamii Maiden et Cambage.

O E. benthamii é originário da Austrália, região de Nova Gales do Sul. Árvore alta, variando de 30 a $45 \mathrm{~m}$ de altura, tem sua distribuição natural restrita ao oeste da cidade de Sydney, em planícies ao longo do rio Nepean e seus afluentes (FAO, 2004). No Brasil, Graça et al. (1999) afirmam que uma das primeiras populações experimentais de $E$. benthamii foi plantada há quase 25 anos, em Colombo, PR, pela Embrapa Florestas, com procedência da Austrália.

Nisgoski et al. (1998) descrevem as características gerais da madeira de E. benthamii como: densidade média (densidade básica de $0,47 \mathrm{~g} / \mathrm{cm}^{3}$ ), cerne e alburno distintos, sendo que o cerne é de cor marrom avermelhada e o alburno amarelo rosado, textura fina a média, grã irregular, cheiro e gosto imperceptíveis, superfície levemente brilhante em luz adequada e anéis de crescimento indistintos. Higa e Pereira (2003) indicam que a madeira de E. benthamii é dimensionalmente instável, apresentando índices elevados de retratibilidade volumétrica e de coeficiente de anisotropia, o que pode resultar em defeitos na produção de madeira serrada.

O objetivo do estudo foi avaliar tecnicamente a produção de painéis de partículas orientadas (OSB) com madeira Eucalyptus benthamii e Pinus spp combinadas em diferentes camadas, a fim de verificar o potencial do Eucalyptus sp em painéis puros e com o mix das espécies.

\section{MATERIAL E MÉTODOS}

O material de E. benthamii foi fornecido em forma de toras pela empresa Klabin, sendo proveniente de reflorestamentos com 13 anos de idade localizados na cidade de Palmeira, SC, as quais possuíam comprimento de $2,40 \mathrm{~m}$ e diâmetro variando entre 20 e $30 \mathrm{~cm}$. No estudo foram utilizadas 20 toras que foram desdobradas por meio do sistema tangencial em peças de madeira serrada de $2,40 \mathrm{~m}$ de comprimento, $80 \mathrm{~mm}$ de largura e $25 \mathrm{~mm}$ de espessura. Estas peças foram seccionadas em peças com $8 \mathrm{~cm}$ de comprimento, sendo posteriormente imersas em um tambor com água à temperatura ambiente durante 7 dias para facilitar o processo de geração das partículas strand. Após a geração, as partículas foram classificadas manualmente para eliminação de partículas irregulares e através de amostragem foram obtidas as dimensões médias de 80 × $25 \times 0,50 \mathrm{~mm}$.

Já o outro material utilizado no estudo foi constituído por partículas tipo strand de $P$. taeda e $P$. elliottii, sendo um mix de $80 \%$ e $20 \%$ respectivamente de cada espécie, o qual foi fornecido pela empresa Louisiana Pacific (LP), Unidade de Ponta Grossa, PR. As dimensões médias de 115 x 27 x $0,70 \mathrm{~mm}$ foram determinadas após a medição de algumas amostras das partículas.

Os painéis foram produzidos com proporções de camadas de 30:40:30 (face:miolo:face) usando uma caixa formadora para orientação das partículas, produzida a partir do modelo de Mendes (2001). A densidade nominal adotada foi de $0,65 \mathrm{~g} / \mathrm{cm}^{3}$ e dimensões do painel de $49,0 \times 42,0 \times 1,50 \mathrm{~cm}$. As partículas foram secas em estufa a temperatura de $80{ }^{\circ} \mathrm{C}$ até atingirem teor de umidade aproximado de 4\%. A resina utilizada foi fenol-formaldeído, que segundo o fabricante, apresentava teor de sólidos de $51,16 \%$ e viscosidade de $480 \mathrm{cp}$. A quantidade de resina aplicada foi de $6 \%$, com base no peso seco das 
partículas, além do uso de $1 \%$ de emulsão de parafina, com 48,62\% de teor de sólidos. Posteriormente fez-se a prensagem a frio com $5 \mathrm{kgf} / \mathrm{cm}^{2}$ e em seguida a prensagem a quente em temperatura de $180^{\circ} \mathrm{C}$ e pressão específica de $40 \mathrm{kgf} / \mathrm{cm}^{2}$ durante 8 minutos.

O delineamento do experimento envolveu a produção de painéis OSB compostos por partículas de E. benthamii e mix de partículas de $P$. taeda e $P$. elliottii, compostos por 3 camadas posicionadas perpendicularmente entre si, comparando-se assim, painéis puros de cada gênero e também combinando Eucalyptus e Pinus em estruturas diferentes de colchão, ora na superfície, ora no miolo, além de camadas com mistura de ambas as espécies, conforme tabela 1.

Tabela 1. Delineamento experimental.

Table 1. Experimental plan.

\begin{tabular}{lccc}
\hline Tratamento & $\begin{array}{c}\text { Composição das camadas } \\
\text { (face:miolo:face) }\end{array}$ & $\begin{array}{c}\text { Proporção das camadas } \\
\text { (face:miolo:face) }\end{array}$ & Número de painéis \\
\hline T1 & $\mathrm{P}: \mathrm{P}: \mathrm{P}$ & $30: 40: 30$ & 3 \\
$\mathrm{~T} 2$ & $\mathrm{E}: \mathrm{E}: \mathrm{E}$ & $30: 40: 30$ & 3 \\
$\mathrm{~T} 3$ & $\mathrm{P}: \mathrm{E}: \mathrm{P}$ & $30: 40: 30$ & 3 \\
$\mathrm{~T} 4$ & $\mathrm{E}: \mathrm{P}: \mathrm{E}$ & $30: 40: 30$ & 3 \\
$\mathrm{~T} 5$ & $\mathrm{P}+\mathrm{E}: \mathrm{P}+\mathrm{E}: \mathrm{P}+\mathrm{E}$ & $30: 40: 30$ & 3 \\
\hline
\end{tabular}

P: Pinus spp. E: E. benthamii. P+E: mistura de Pinus spp. e E. benthamii.

Após o processo de produção dos painéis, procedeu-se o esquadrejamento e a retirada dos corpos de prova, sendo armazenados em uma sala de climatização à temperatura de $20 \pm 2{ }^{\circ} \mathrm{C}$ e umidade relativa de $65 \pm 3 \%$ até atingirem massa constante. Os ensaios físicos foram realizados de acordo com a ASTM 1037 (1993), sendo eles: densidade, teor de umidade, absorção de água, inchamento em espessura, taxa de não retorno em espessura, e ainda, o ensaio mecânico de ligação interna. Já para a propriedade mecânica de flexão estática, a norma adotada foi a DIN 52362 (1982) em função do menor comprimento do corpo de prova, o que corrobora com as dimensões do painel produzido no laboratório.

Para razão de compactação, que relaciona densidade do painel e densidade da madeira, foi necessária a determinação da densidade da madeira das duas espécies. Para o E. benthamii, a cada 30 peças que eram obtidas no processo de seccionamento das peças de madeira serrada (processo descrito anteriormente), se retirava uma peça para a determinação da densidade, totalizando 56 corpos de prova. Assim, a densidade do E. benthamii foi determinada de acordo com os procedimentos da NBR 11941 (2003). Já a densidade do mix de Pinus spp foi determinada pelo valor médio de trabalhos científicos, os quais estudaram as espécies em diversas idades e de diversos locais, tendo em vista que a empresa não forneceu madeira sólida para a determinação da variável.

Após os ensaios laboratoriais, os dados foram submetidos a análises preliminares, nas quais foram verificados os pressupostos para utilização da estatística paramétrica. Assim, os dados foram testados quanto à presença de outliers, normalidade da distribuição e homogeneidade de variâncias. $\mathrm{O}$ primeiro teste foi realizado por meio de análise gráfica (boxplot) e descritiva dos dados, o segundo pelo teste de Kolmogorov-Smirnov, e por fim, as variâncias foram avaliadas por meio do teste de Levene.

Com os pressupostos atendidos, os dados foram analisados estatisticamente por meio da Análise de Variância e havendo a rejeição da hipótese de igualdade, foi aplicado o Teste de Scott-Knott a 95\% de probabilidade. Além da análise estatística tradicional, os valores médios dos tratamentos foram comparados com as especificações da norma CSA 0437 (1993).

\section{RESULTADOS E DISCUSSÃO}

\section{Propriedades físicas}

Os tratamentos não apresentaram diferença significativa para os valores médios de densidade dos painéis, conforme tabela 2, sendo classificados como de média densidade de acordo com Iwakiri (2005) que estabelece intervalo entre 0,59 a $0,80 \mathrm{~g} / \mathrm{cm}^{3}$ para tal categoria.

Em relação à razão de compactação, somente o tratamento T1 composto exclusivamente por partículas de E. benthamii não ficou dentro do intervalo recomendado por Maloney (1977) para que os painéis apresentem boa resistência mecânica, que é de 1,30 a 1,60.

FLORESTA, Curitiba, PR, v. 45, n. 4, p. 865 - 874, out. / dez. 2015.

Gorski, L. et al.

ISSN eletrônico 1982-4688 / ISSN impresso 0015-3826

DOI: $10.5380 /$ rf.v45i4.36280 
Tabela 2. Valores médios para densidade do painel, razão de compactação e teor de umidade.

Table 2. Medium values for panel density, compression ratio and moisture content.

\begin{tabular}{llcccc}
\hline Tratamento & & DP $\left(\mathbf{g} / \mathbf{c m}^{\mathbf{3}}\right)$ & $\mathbf{R C}$ & Espessura $(\mathbf{m m})$ & TU $(\%)$ \\
\hline T1 & E:E:E & $0,66_{(8,12)} \mathrm{a}$ & $1,25_{(8,12)} \mathrm{b}$ & $17,04_{(12,43)} \mathrm{a}$ & $10,10_{(7,24)} \mathrm{b}$ \\
T2 & P:P:P & $0,64_{(5,40)} \mathrm{a}$ & $1,52_{(5,40)} \mathrm{a}$ & $17,86_{(18,62)} \mathrm{b}$ & $10,40_{(4,87)} \mathrm{a}$ \\
T3 & E:P:E & $0,64_{(5,62)} \mathrm{a}$ & $1,32_{(5,62)} \mathrm{b}$ & $17,23_{(13,05)} \mathrm{a}$ & $09,80_{(9,38)} \mathrm{c}$ \\
T4 & P:E:P & $0,67_{(6,02)} \mathrm{a}$ & $1,47_{(6,02)} \mathrm{a}$ & $17,47_{(16,19)} \mathrm{a}$ & $09,90_{(10,04)} \mathrm{c}$ \\
T5 & P+E:P+E:P+E & $0,64_{(8,82)} \mathrm{a}$ & $1,37_{(8,82)} \mathrm{b}$ & $17,31_{(15,96)} \mathrm{a}$ & $10,00_{(8,38)} \mathrm{b}$ \\
\hline Média & & 0,65 & 1,38 & 17,32 & 10,04 \\
\hline
\end{tabular}

Legenda: E: E. benthamii; P: Mix de P. taeda e P. elliottii. DP: densidade dos painéis. RC: razão de compactação. TU: teor de umidade. Subscrito: coeficiente de variação (\%). Médias seguidas pela mesma letra na coluna não diferem estatisticamente entre si, a $95 \%$ de probabilidade, pelo teste de Scott-Knott.

Os resultados variaram de 1,25 (T1 - E:E:E) até 1,52 (T2 - P:P:P) apresentando diferença estatística significativa. Observa-se que os valores médios dos tratamentos compostos totalmente (100\%) ou em grande quantidade $(60 \%)$ por madeira de Pinus, possuem maior razão de compactação que os tratamentos formados por madeira de Eucalyptus. Assim, alguns autores explicam estes resultados pelo fato da madeira de Pinus apresentar baixa densidade o que resulta em painéis de alta razão de compactação e maior área de contato entre as partículas, influenciando positivamente nas propriedades de flexão estática e ligação interna (MALONEY, 1993).

Em relação à espessura dos painéis, observa-se que houve diferença estatística do tratamento T2 e os demais. Mesmo assim, todos os valores médios de espessura ficaram acima dos espaçadores da prensa $(16 \mathrm{~mm})$. Acredita-se que o fato da espessura dos painéis de todos os tratamentos estarem acima do desejado, se deva ao grande volume de partículas no colchão para atingir a densidade nominal, o que tem como consequência uma maior liberação parcial das tensões de compressão após o final da prensagem na fase de produção dos painéis. Todavia, este efeito foi ainda mais pronunciado no tratamento T2 que é composto exclusivamente por partículas de Pinus spp (T2) que tem densidade de $0,41 \mathrm{~g} / \mathrm{cm}^{3}$, ou seja, menor que a do E. benthamii que possui densidade de $0,53 \mathrm{~g} / \mathrm{cm}^{3}$.

Para os valores médios de teor de umidade, observa-se variação entre $9,8 \%$ e $10,4 \%$ com baixo coeficiente de variação dentro de cada tratamento, entretanto, houve diferença estatística entre os tratamentos. Resultados de teor de umidade semelhantes foram obtidos por Del Menezzi (2004) que encontrou 9,27\% e Mendes (2010) que encontrou média de $8 \%$ para painéis OSB de Pinus. Já para painéis de Eucalyptus, Okino (2008) obteve 8\% de teor de umidade. Em escala comercial, a empresa produtora de painéis OSB no Brasil adota uma faixa aceitável de 2 a $12 \%$ de umidade em seus produtos finais.

A tabela 3 apresenta os valores médios para absorção em água, inchamento em espessura e taxa de não retorno em espessura para os painéis OSB, onde podem ser observados valores elevados das propriedades, bem como a diferença estatística entre os tratamentos.

Tabela 3. Valores médios para absorção de água e inchamento em espessura, após 2 e 24 horas de imersão em água, e taxa de não retorno em espessura.

Table 3. Medium values of water absorption and thickness swelling, 2 and 24 hours after immersion in water, and irreversible thickness swelling rate.

\begin{tabular}{llccccc}
\hline \multirow{2}{*}{ Tratamento } & & AA 2h & AA 24h & IE 2h & IE 24h & TNRE \\
\cline { 3 - 7 } & & \multicolumn{7}{c}{$\mathbf{c h}$} \\
\hline T1 & E:E:E & $18,3_{(22,68)} \mathrm{a}$ & $54,2_{(13,54)} \mathrm{a}$ & $09,1_{(18,40)} \mathrm{a}$ & $24,7_{(14,00)} \mathrm{a}$ & $26,7_{(12,02)} \mathrm{a}$ \\
T2 & P:P:P & $62,6_{(17,77)} \mathrm{d}$ & $95,2_{(12,71)} \mathrm{d}$ & $24,6_{(15,92)} \mathrm{d}$ & $32,8_{(09,35)} \mathrm{b}$ & $36,6_{(07,01)} \mathrm{b}$ \\
T3 & E:P:E & $26,7_{(27,77)} \mathrm{b}$ & $68,4_{(24,74)} \mathrm{b}$ & $13,1_{(24,87)} \mathrm{b}$ & $28,3_{(19,78)} \mathrm{a}$ & $29,0_{(13,82)} \mathrm{a}$ \\
T4 & P:E:P & $48,3_{(22,58)} \mathrm{c}$ & $79,4_{(24,30)} \mathrm{c}$ & $18,8_{(18,73)} \mathrm{c}$ & $28,7_{(16,93)} \mathrm{a}$ & $31,6_{(12,76)} \mathrm{a}$ \\
T5 & P+E:P+E:P+E & $27,0_{(29,73)} \mathrm{b}$ & $80,2_{(16,31)} \mathrm{c}$ & $16,9_{(21,81)} \mathrm{c}$ & $32,0_{(20,06)} \mathrm{b}$ & $36,2_{(12,97)} \mathrm{b}$ \\
\hline Média & & 36,57 & 75,45 & 16,52 & 32,00 & 29,31 \\
\hline
\end{tabular}

Legenda: E: E. benthamii; P: Mix de P. taeda e P. elliottii. AA: absorção de água. IE: inchamento em espessura. TNRE: taxa de não retorno em espessura. CV: coeficiente de variação. Subscrito: coeficiente de variação (\%). Médias seguidas pela mesma letra na coluna não diferem estatisticamente entre si, a $95 \%$ de probabilidade, pelo teste de Scott-Knott. 
Os resultados para absorção de água após 2 e 24 horas e inchamento em espessura após 2 horas de imersão seguiram a mesma tendência, onde os melhores resultados, isto é, as menores médias, foram obtidas pelo tratamento 1 (E:E:E) e o pior desempenho foi observado para o tratamento 2 (P:P:P). Para inchamento em espessura 24 horas e taxa de não retorno em espessura, os menores valores médios também foram observadas para o T1 embora não tenha diferido estatisticamente dos tratamentos T3 e T4.

A absorção de água, diferentemente do teor de umidade de equilíbrio, é um fenômeno muito mais físico que químico e está relacionado à porosidade (espaços vazios) dos painéis e da madeira propriamente dita. Quando o painel está submerso, a água líquida ocupa os espaços vazios formados entre as partículas e o interior das células da madeira, onde permanece pela ação de forças capilares, conforme Del Menezzi (2004).

A norma CSA 0437 (1993) exibe apenas o valor para inchamento em espessura após 24 horas de imersão em água, sendo que deve ser no máximo de $15 \%$ para painéis de $12,7 \mathrm{~mm}$ de espessura ou menores e $10 \%$ para painéis superiores a $12,7 \mathrm{~mm}$ de espessura. Assim, observa-se que nenhum tratamento foi satisfatório em relação, apresentando, em média, o dobro do requisitado (32\%). Este problema é frequente se observado outros autores que trabalharam com $6 \%$ de resina fenol formaldeído, como Surdi (2012) que obteve média de 37,95\% (Pinus sp.); Gouveia et al. (2003) que obtiveram 25,81\% (painéis com $P$. elliottii e $E$. grandis) e Iwakiri et al. (2004) que encontraram resultados variando de $28,85 \%$ (E. grandis) a 70,96\% (E. dunnii).

Kelly (1977) diz que quanto maior a razão de compactação, maior será o inchamento residual. Neste estudo isto também foi observado, já que os painéis compostos por Pinus alcançaram o maior valor médio de taxa de não retorno em espessura $(36,6 \%)$, possuindo também a maior razão de compactação $(1,52)$. Mendonça (2008) afirma que isto se deve à maior concentração de material lenhoso dos painéis com maior RC que, consequentemente, resultam em maior absorção de água e inchamento em espessura.

Para clones de Eucalyptus, Mendes et al. (2007) encontrou valor bastante inferior (7,27\%), entretanto, ressalta-se o alto coeficiente de variação que a variável apresentou. Mendonça (2008) analisando clones de E. urophylla encontrou valores de taxa de não retorno em espessura variando de 14,61\% a 20,67\%. Já Mendes (2001) obteve médias entre 14,61\% (P. oocarpa) e 24,39\% (mistura de 6 espécies de Pinus).

\section{Propriedades mecânicas}

De acordo com a tabela 4, evidencia-se que os valores médios dos tratamentos para módulo de ruptura (MOR) paralelo variaram de 26,6 a 51,5 MPa e perpendicular de 15,3 a 30,4 MPa, demonstrando o efeito da orientação das partículas. O mesmo pode ser observado para o módulo de elasticidade (MOE), onde no sentido paralelo o intervalo foi entre 4368,9 e 7917,5 MPa e no sentido perpendicular entre 1194,2 a 1820,6 MPa.

Tabela 4. Valores médios para módulo de ruptura e módulo de elasticidade (paralelo e perpendicular).

Table 4. Medium values of modulus of rupture and modulus of elasticity (parallel and perpendicular).

\begin{tabular}{|c|c|c|c|c|c|}
\hline \multirow{2}{*}{\multicolumn{2}{|c|}{ Tratamento }} & $\begin{array}{c}\text { MOR } \\
\text { paralelo }\end{array}$ & $\begin{array}{c}\text { MOE } \\
\text { paralelo }\end{array}$ & $\begin{array}{c}\text { MOR } \\
\text { perpendicular }\end{array}$ & $\begin{array}{c}\text { MOE } \\
\text { perpendicular }\end{array}$ \\
\hline & & \multicolumn{4}{|c|}{ (MPa) } \\
\hline T1 & E:E:E & $26,6_{(19,05)} b$ & $4368,0_{(26,47)} b$ & $15,3_{(17,54)} b$ & $1194,2_{(29,00)} b$ \\
\hline $\mathrm{T} 2$ & P:P:P & $43,3_{(08,58)}$ a & $4531,9_{(17,84)} b$ & $30,4_{(10,84)}$ a & $1762,2_{(20,09)}$ a \\
\hline $\mathrm{T} 3$ & $\mathrm{E}: \mathrm{P}: \mathrm{E}$ & $51,5_{(25,64)}$ a & $7917,5_{(34,78)} \mathrm{a}$ & $27,1_{(31,32)}$ a & $1791,1_{(31,30)}$ a \\
\hline $\mathrm{T} 4$ & $\mathrm{P}: \mathrm{E}: \mathrm{P}$ & $42,1_{(14,40)} \mathrm{a}$ & $4690,6_{(29,12)} b$ & $30,3_{(11,73)}$ a & $1820,6_{(30,04)}$ a \\
\hline T5 & $\mathrm{P}+\mathrm{E}: \mathrm{P}+\mathrm{E}: \mathrm{P}+\mathrm{E}$ & $38,9_{(20,63)}$ a & $5825,3_{(31,64)} b$ & $20,3_{(22,26)} b$ & $1428,4_{(30,73)} b$ \\
\hline Média & & 40,49 & 5466,66 & 24,66 & 1599,32 \\
\hline
\end{tabular}

No sentido perpendicular, tanto para MOR quanto para MOE, houve diferença estatística entre os tratamentos, embora ambas as propriedades tenham seguido uma tendência, onde os melhores resultados foram obtidos nos tratamentos T2 (P:P:P), T3 (E:P:E) e T4 (P:E:P). No sentido paralelo, para MOE, o tratamento com melhor desempenho foi o T3 (E:P:E) com média de 7917,5 MPa, bem mais

FLORESTA, Curitiba, PR, v. 45, n. 4, p. 865 - 874, out. / dez. 2015.

Gorski, L. et al.

ISSN eletrônico 1982-4688 / ISSN impresso 0015-3826

869

DOI: $10.5380 /$ rf.v45i4.36280 
elevada que os demais. Já para MOR, no sentido paralelo, o tratamento T3 (E:P:E) também se destacou com a maior média, entretanto, não diferiu dos demais, exceto do tratamento T1 (E:E:E) que obteve a menor média entre todos os tratamentos, 26,6 MPa.

Moslemi (1974) observou que painéis produzidos com espécies de baixa densidade geralmente apresentam maior resistência à flexão e a tração, melhor módulo de elasticidade e melhor ligação interna do que os painéis obtidos de espécies de alta densidade para painéis de igual densidade. Assim, a escolha do tipo de madeira é função do uso final do produto e vice-versa. Os resultados estão de acordo com o afirmado pelo autor, já que o tratamento com Pinus foi maior em todas as propriedades mecânicas, quando comparado ao tratamento composto exclusivamente de Eucalyptus.

Os parâmetros da norma CSA 0437 (1993) definem valores mínimos de resistência e rigidez de: MOR paralelo e perpendicular de 23,4 e 9,6 MPa, MOE paralelo e perpendicular de 4500 e $1300 \mathrm{MPa}$ (classe O-1); MOR paralelo e perpendicular de 29,0 e 12,4 MPa, MOE paralelo e perpendicular de 5.500 e $1.500 \mathrm{MPa}$ (classe $\mathrm{O}-2$ ). Assim, todos os tratamentos atingiram as exigências da norma ou para a classe O-2 ou O-1, exceto o tratamento T1 (E:E:E) que não atingiu nem as especificações da classe menos exigente para o módulo de elasticidade nos dois sentidos.

Comparativamente, pode-se citar outros autores. Mendes et al. (2012) encontraram para painéis OSB de $P$. oocarpa os valores médios de MOE perpendicular às fibras variando de 1079 a $1772 \mathrm{MPa}$, enquanto que o MOR variou de 10 a $23 \mathrm{MPa}$. Já os valores de MOE paralelo às fibras variaram de 4782 a $6333 \mathrm{MPa}$ e o MOR paralelo variou de 41 a $63 \mathrm{MPa}$. Estes resultados são inferiores aos obtidos no presente estudo para o sentido perpendicular e superiores para o sentido paralelo. Nota-se ainda, que os resultados obtidos pelo autor no sentido paralelo também foram maiores que no sentido perpendicular. Valores inferiores em relação a este estudo foram obtidos por Mendes et al. (2007), trabalhando com OSB de clones de Eucalyptus e resina fenólica, que encontraram valores médios de MOE paralelo igual a $3108 \mathrm{MPa}$ e $958 \mathrm{MPa}$ para MOE perpendicular. Para MOR paralelo o resultado foi de $25 \mathrm{MPa}$ e $12 \mathrm{MPa}$ para o perpendicular.

A relação positiva entre densidade e propriedades mecânicas não foi encontrada para os tratamentos avaliados, no entanto houve relação direta entre essas propriedades e a razão de compactação dos painéis. Afinal, a maior razão de compactação foi obtida pelo tratamento T2 com P:P:P $(1,52)$ e a menor pelo tratamento T1 com E:E:E $(1,25)$.

Como mostra a tabela 5 , houve diferença significativa entre os tratamentos para ligação interna, apontando para um melhor desempenho dos tratamentos T2 e T3, que, diferiram estatisticamente dos demais. A média geral obtida para este ensaio foi de 0,56 MPa.

Tabela 5. Valores médios de ligação interna.

Table 5. Medium values of internal bond.

\begin{tabular}{lcc}
\hline \multicolumn{1}{l}{ Tratamento } & \multicolumn{1}{c}{ LI } \\
\hline T1 & E:E:E & $0,50_{(07,59)} \mathrm{b}$ \\
T2 & P:P:P & $0,67_{(04,56)} \mathrm{a}$ \\
T3 & E:P:E & $0,63_{(15,48)} \mathrm{a}$ \\
T4 & P:E:P & $0,50_{(11,15)} \mathrm{b}$ \\
T5 & P+E:P+E:P+E & $0,52_{(14,06)} \mathrm{b}$ \\
\hline Média & & 0,56 \\
\hline E: $E$. benthamii; P: Mix de $P$. taeda e $P$. elliottii. LI: ligação interna. CV: coeficiente de \\
variação. Subscrito: coeficiente de variação (\%). Médias seguidas pela mesma letra na coluna \\
não diferem estatisticamente entre si, a 95\% de probabilidade, pelo teste de Scott-Knott.
\end{tabular}

O melhor desempenho, em termos numéricos, da ligação interna dos painéis formados por partículas de Pinus pode estar relacionado à maior porosidade da madeira da espécie em relação ao Eucalyptus, que favorece o espalhamento e a penetração da resina. Iwakiri (2005) afirma que o desempenho da colagem da madeira está intrinsicamente vinculada à porosidade e à permeabilidade.

Outro fato que pode ter colaborado para a superioridade da colagem do tratamento P:P:P é o tamanho maior das partículas de Pinus spp. $(115 \times 27 \times 0,70 \mathrm{~mm})$ em relação às de E. benthamii $(80 \times 25 \times 0,50 \mathrm{~mm})$. Maloney (1993) afirma que com a aplicação da mesma quantidade de resina, o painel produzido com partículas maiores apresentará maior resistência a ligação interna, devido sua 
menor área superficial específica e, consequentemente, maior disponibilidade de resina por unidade de área.

Os resultados de ligação interna atingiram o valor mínimo exigido pela norma CSA 0437 (1993) que é de $0,345 \mathrm{MPa}$ mostrando que a colagem foi bem realizada. Com relação a outros trabalhos, observa-se inferioridade de valores médios em relação à Gouveia (2001), que encontrou para painéis com P. elliottii 0,77 MPa, e equivalência com Iwakiri et al. (2004) que obtiveram para painéis com E. grandis, $0,57 \mathrm{MPa}$.

De acordo Iwakiri (1989), o teste de ligação interna expressa o grau de adesão entre as partículas e expõe onde está localizada a superfície de maior fragilidade. Assim, a ruptura ocorre geralmente na porção mediana de sua espessura, a qual corresponde à região de menor densidade e ligação entre as partículas. No entanto, este fato, muitas vezes não foi observado nos ensaios, onde a ruptura acabava acontecendo entre as camadas do painel, principalmente nos tratamentos T3 e T4 que apresentam camadas formadas por espécies diferentes. Esta situação também foi encontrada por Gouveia et al. (2003) na colagem de painéis formados por espécies diferentes (Pinus spp e Eucalyptus spp)., atribuindo o comportamento pela diferença de densidade das espécies e diferença de absorção do adesivos pelas mesmas.

Assim como a espécie, outros fatores interferem nos resultados finais da colagem de partículas. Mendes (2001) verificou aumento nos valores de ligação interna com o aumento na densidade dos painéis OSB de Pinus. Este fato não foi constatado no presente estudo, haja vista que as maiores médias de ligação interna foram obtidas nos tratamentos T3 (P:P:P) e T4 (E:P:E), 0,67 MPa e 0,63 MPa, respectivamente. Estes apresentaram as menores densidades de painéis, entretanto, ressalta-se que a baixa amplitude dos resultados, por consequência, não resultou em diferença estatística para densidade dos painéis o que pode ter sido a causa da não influência sobre a ligação interna.

Pode-se relacionar positivamente o maior resultado de ligação interna $(0,67 \mathrm{MPa})$ com a maior razão de compactação $(1,52)$ do tratamento T2 (P:P:P) e também, o menor resultado de ligação interna $(0,50 \mathrm{MPa})$ com a menor razão de compactação $(1,25)$ do tratamento T1 (E:E:E). Os painéis com alta razão de compactação possuem uma maior área de contato entre as partículas, resultando em melhores propriedades de ligação interna (MALONEY, 1993).

\section{CONCLUSÕES}

- Os resultados obtidos no presente estudo permitiram avaliar a qualidade dos painéis quanto:

- a norma de referência: para a estabilidade dimensional dos painéis (imersão 24 horas), nenhum tratamento atendeu ao estabelecido; já em relação às propriedades de flexão estática e ligação interna, os painéis podem ser classificados como O-2, exceção feita ao tratamento T1 (E:E:E) que não atendeu aos parâmetros.

- ao desempenho: as melhores composições 30:40:30 foram obtidas nos tratamentos T2 composto exclusivamente por partículas de Pinus spp e T3 com partículas de Pinus spp na camada central e $E$. benthamii nas camadas externas.

- da utilização da madeira de E. benthamii na produção de painéis OSB: foi observado potencial da espécie para a produção dos painéis quando misturada com partículas de $P$. taeda e $P$. eliiottii, as quais são utilizadas tradicionalmente pelo setor. Na composição pura, os painéis com E. benthamii apresentam resultados de resistência e rigidez insatisfatórios

\section{AGRADECIMENTOS}

As empresas LP Brasil, Klabin S.A., Madepar Indústria e Comércio de Madeiras LTDA, SI Group Crios Resinas e a Indústria de Compensados Sudati LTDA. Ao SENAI - Unidade Lages.

\section{REFERÊNCIAS}

AMERICAN SOCIETY FOR TESTING AND MATERIALS (ASTM). ASTM D-1037. Standard methods of evaluating properties of wood-base fiber and particles materials. Philladelphia: Annual book of ASTM Standard, 1993.

FLORESTA, Curitiba, PR, v. 45, n. 4, p. 865 - 874, out. / dez. 2015.

Gorski, L. et al.

ISSN eletrônico 1982-4688 / ISSN impresso 0015-3826

871

DOI: $10.5380 /$ rf.v45i4.36280 
ASSOCIAÇÃO BRASILEIRA DE NORMAS TÉCNICAS (ABNT). Normas técnicas NBR 11941: Madeira - determinação da densidade básica. Rio de Janeiro, 2003, p. 06.

CABRAL, C. P. T.; VITAL, B. R.; LUCIA, R. M. D.; PIMENTA, A. S.; SOARES, C. P. B.; CARVALHO, A. M. M. L. Propriedades de chapas tipo OSB, fabricadas com partículas acetiladas de madeiras de Eucalyptus grandis, Eucalyptus urophylla, Eucalyptus cloeziana e Pinus elliottii. Árvore, Viçosa, v. 30, n. 4, p. 659 - 668, 2006.

CASTRO, M. B.; CÉSAR, A. A. da S.; BUFALINO, L.; MENDES, L. M.; MENDES, R. F. Resistência mecânica de painéis OSB de pinus e eucalipto. In: ENCONTRO BRASILEIRO EM MADEIRAS E EM ESTRUTURAS DE MADEIRA. Anais. Vitória, 2012.

CSA. CANADIAN STANDARDS ASSOCIATION. OSB and Waferboard. CSA: 0437. Ontario, 1993. $18 \mathrm{p}$.

DEL MENEZZI, C. H. S. Estabilização dimensional por meio do tratamento térmico e seus efeitos sobre as propriedades de painéis de partículas orientadas (OSB). $242 \mathrm{f}$. Tese (Doutorado em Ciências Florestais) - Universidade Federal do Paraná, Curitiba, 2004.

DIN. NORMEN FÜR HOLZFASERPLATEN SPANPLATTEN SPERRHOLZ. Testing of wood chipboards bending test, determination of bending strength: DIN 52362. Berlin, 1982, $40 \mathrm{p}$.

FAO. Forest genetic resources conservation and management. Vol. 1: Overview, concepts and some systematics approaches. Rome: International Plant Genetic Resources Institute, 2004.

GOUVEIA, F. N. Produção de chapas de partículas orientadas (OSB) a partir das espécies Eucalyptus grandis W. HILL EX MAIDEN e Pinus elliottii Engelm. 81 f. Tese (Doutorado em Ciência Florestal) - Universidade Federal de Viçosa, Viçosa, MG, 2001.

GOUVEIA, F. N.; VITAL, B. R.; SANTANA, M. A. Avaliação de três tipos de estrutura de colchão e três níveis de resina fenólica na produção de chapas de partículas orientadas - OSB. Revista Árvore, Viçosa, v. 27, n. 3, p. 365 - 370, 2003.

GRAÇA, M. E. C.; CARAMORI, P. H.; BOOTSMA, A.; OLIVEIRA, D.; GOMES, J. Capacidade de rebrota e de enraizamento de Eucalyptus benthamii. Boletim de Pesquisa Florestal, Colombo, n. 39, p. $135-138,1999$.

HIGA, R. C. V.; PEREIRA, J. C. D. Usos potenciais do Eucalyptus benthamii Maiden et Cambage. (Comunicado Técnico n. 100). Colombo: Embrapa Florestas. 2003, 4 p.

IWAKIRI, S. Painéis de madeira reconstituída. Curitiba: FUPEF, 2005, p. 123 - 160.

IWAKIRI, S.; MENDES, L. M.; SALDANHA, L. K.; SANTOS, J. C. dos. Utilização da madeira de eucalipto na produção de chapas de partículas orientadas - OSB. Cerne, Lavras, v. 10, n. 1, p. 46 - 52, 2004.

IWAKIRI, S. A. influência de variáveis de processamento sobre propriedades de chapas de partículas de diferentes espécies de Pinus. $129 \mathrm{f}$. Tese (Doutorado em Ciências Florestais) Universidade Federal do Paraná, Curitiba, 1989.

KELLY, M. W. A critical literature review of relationships between processing parameters and physical properties of particleboards. Forest Products Laboratory - Lab. General Technical Report (FPL-10), Madison, 1977. $66 \mathrm{p}$.

MALONEY, T. M. Modern particleboard e dry-process fiberboard manufacturing. São Francisco: Miller Freeman Publication, 2 ed. 1993. 689 p.

MALONEY, T. M. Modern Particleboard \& Dry Process Fiberboard manufacturing. San Francisco: Miller Freeman Publication, 1977, 672 p.

MENDES, R. F.; MENDES, L. M.; CARVALHO, A. G.; SILVA, F. A. da; GUIMARÃES JÚNIOR, J. B. Ciência da Madeira, Pelotas, v. 3, n. 2, p. 116 - 127, 2012. 
MENDES, R. F. Efeito do tratamento térmico sobre as propriedades de painéis OSB. $115 \mathrm{f}$. Dissertação (Mestrado em Ciências Florestais) - Escola Superior de Agricultura "Luiz de Queiroz", USP, Piracicaba, 2010.

MENDES, S. A.; MENDES, L. M.; CHAVES, M. D.; MORI, F. A.; SILVA, J. R. M.; TRUGILHO, P. F. Utilização de resinas alternativas na produção de painéis OSB de clones de Eucalyptus spp. Cerne, Lavras, v. 13, n. 3, p. 257 - 263, 2007.

MENDES, L. M. Pinus spp. na produção de painéis de partículas orientadas (OSB). 103 f. Tese (Doutorado em Ciências Florestais) - Universidade Federal do Paraná, Curitiba, 2001.

MENDONÇA, L. L. Produção de painéis de madeira com clones de Eucalyptus urophylla: estudo de caso para uso na indústria de móveis e construção civil. 129 f. Dissertação (Mestrado em Engenharia de Materiais) - REDEMAT (UFOP - CETEC - UEMG), 2008.

MOSLEMI, A. A. Particleboard: technology. London: Southern Illinois University, 2 v. 1974, 245 p.

NISGOSKI, S.; MUNIZ, G. I. B.; KLOCK, U. Caracterização anatômica da madeira de Eucalyptus benthamii Mainden et Cambage. Ciência Florestal, Santa Maria, v. 8, n. 1, p. 67 - 76, 1998.

OKINO, E. Y. A., Propriedades de chapas OSB de Eucalyptus grandis e de Cupressus glauca. Scientia Forestalis, Piracicaba, v. 36, n. 78, p. 123 - 131, 2008.

SURDI, P. G. Produção de painéis de partículas orientadas (OSB) a partir da madeira de um híbrido de Pinus elliottii var. elliottii X Pinus caribaea var. hondurensis. 98 f. Dissertação (Mestrado em Ciências Florestais) - Escola Superior de Agricultura "Luiz de Queiroz", USP, Piracicaba, Piracicaba, 2012. 